\title{
Healthcare cost of patients with multiple chronic diseases in Singapore public primary care setting
}

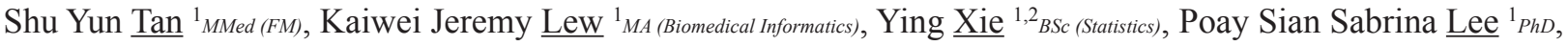

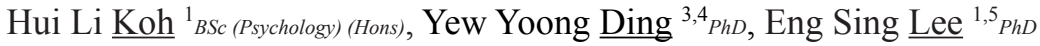

\begin{abstract}
Introduction: The rising prevalence of multiple chronic diseases is an important public health issue as it is associated with increased healthcare utilisation. This paper aimed to explore the annual per capita healthcare cost in primary care for patients with multiple chronic diseases (multimorbidity).

Methods: This was a retrospective cohort study conducted in a cluster of public primary care clinics in Singapore. De-identified data from electronic medical records were extracted from July 2015 to June 2017. Only patients with at least 1 chronic disease were included in the study. Basic demographic data and healthcare cost were extracted. A list of 20 chronic diseases was considered for multimorbidity.

Results: There were 254,377 patients in our study population, of whom $52.8 \%$ were female. The prevalence of multimorbidity was $62.4 \%$. The median annual healthcare cost per capita for patients with multimorbidity was about twice the amount compared to those without multimorbidity (SGD683 versus SGD344). The greatest percentage increment in cost was when the number of chronic diseases increased from 2 to $3(43.0 \%)$.

Conclusion: Multimorbidity is associated with higher healthcare cost in primary care. Since evidence for the optimal management of multimorbidity is still elusive, prevention or delay in the onset of multimorbidity in the general population is paramount.
\end{abstract}

Ann Acad Med Singap 2021;50:809-17

Keywords: Chronic disease, healthcare cost, multimorbidity, primary care

\section{INTRODUCTION}

The rising occurrence of individuals suffering from multiple chronic diseases, namely multimorbidity, is of public health concern globally. ${ }^{1}$ The current prevalence of multimorbidity in Singapore ranges from 26 to $89 \%$ by various studies depending on the definition used and the population studied. ${ }^{2-5}$ With Singapore's fast ageing population, where 1 in 4 individuals will be more than 65 years and older by $2030,{ }^{6}$ the prevalence of multimorbidity in Singapore is expected to increase.

The burden of multimorbidity is associated with increase in healthcare cost. ${ }^{7-10}$ In Canada, the total cost for adults aged 65 years and above increased from CAD1,026 to CAD3,831 when the number of chronic diseases increased from 2 to 5 or more. ${ }^{11}$ In 2017, the Singapore government spent SGD10.7 billion in healthcare $\left(2.5 \%\right.$ of gross domestic product). ${ }^{12}$ This amount is expected to increase by another SGD3 billion in 2020 .

Many patients with multimorbidity are managed in the primary healthcare system. Therefore, it is important to understand the economic impact of increasing multimorbidity in the primary care setting. It has been reported that the estimated annual societal cost per capita of multimorbidity ( 2 or more chronic diseases) in Singapore was SGD15,148 and the direct medical cost to public primary care was SGD303. ${ }^{13}$ In another study, the annual cost per capita to public primary care (with

\footnotetext{
${ }^{1}$ Clinical Research Unit, National Healthcare Group Polyclinics, Singapore

${ }^{2}$ Information Management and Analytics, National Healthcare Group Polyclinics, Singapore

${ }^{3}$ Department of Geriatric Medicine, Tan Tock Seng Hospital, Singapore

${ }^{4}$ Geriatric Education and Research Institute, Singapore

${ }^{5}$ Lee Kong Chian School of Medicine, Nanyang Technological University, Singapore

Correspondence: Dr Eng Sing Lee, Clinical Research Unit, National Healthcare Group Polyclinics, 3 Fusionopolis Link, Nexus@One-North \#06-13, South Lobby, Singapore 138543.

Email: Eng_Sing_LEE@nhgp.com.sg
} 


\section{CLINICAL IMPACT}

\section{What is New}

- This study characterises the healthcare costs attributable to multimorbidity, and the costs of various combinations of chronic diseases in the Singapore public primary healthcare system.

\section{Clinical Implications}

- Multimorbidity presents a significant cost burden to patients and the healthcare system.

- The cost of the top 10 most prevalent triads (3 chronic diseases) in this study will provide information to further research in the prevention or delay in the onset of these chronic diseases.

2 chronic diseases) was in the range of SGD317.95 to SGD694.71. ${ }^{4}$ However, there are knowledge gaps in the literature about the impact of multimorbidity on primary healthcare cost in Singapore. For example, the incremental primary healthcare cost with increasing number of chronic diseases is currently unknown in Singapore. Moreover, current studies of healthcare costs in the Singapore population are limited in the following ways.

Firstly, results based on patient survey on chronic diseases and service utilisation were limited by recall bias. ${ }^{13}$ Secondly, high prevalent diseases such as hyperlipidaemia, obesity and chronic kidney diseases were not included in the list of multimorbidity diseases. ${ }^{5}$ Thirdly, some existing studies restricted the list of chronic diseases to less than 12 diseases for multimorbidity, where fewer than 12 diseases were found not to be suitable in multimorbidity studies. ${ }^{1}$ Fourthly, different methods and components were used to compute primary care healthcare cost with details of the components missing., ${ }^{413}$ Finally, all of the studies of healthcare costs in the Singapore population defined multimorbidity as 2 or more chronic diseases. However, using 3 or more chronic diseases is clinically more meaningful than using 2 or more chronic diseases as the higher cut-off helps to identify a smaller number of patients with higher needs. ${ }^{14}$

Therefore, in this study, our primary aim was to determine the annual healthcare cost per capita incurred by patients with chronic diseases. Other secondary aims were to (1) determine the incremental cost for patients with increasing number of chronic diseases; (2) compare the healthcare cost incurred by patients with and without multimorbidity; and (3) determine the annual healthcare cost per capita of the 10 most prevalent triads ( 3 chronic diseases) in primary care.

\section{METHODS}

\section{Singapore primary healthcare system}

Singapore has a unique healthcare system that provides universal health coverage with a variety of financial systems, which range from various government insurance schemes and subsidies to private insurance. ${ }^{15}$ The country's public healthcare system offers primary, secondary and tertiary care in 3 different geographic regions.

Primary care is provided by 20 government-subsidised polyclinics and 1,700 private general practitioner (GP) clinics. Polyclinics serve as one-stop centres that provide preventive, acute and chronic healthcare services such as health screening, immunisation, outpatient care, maternal and child healthcare, diagnostic, laboratory and pharmacy services. ${ }^{16-18}$ On average, there are about 6 million attendances at the polyclinics annually. ${ }^{19}$ According to the Primary Care Survey 2014, up to $52 \%$ of the visits to polyclinics were for chronic medical diseases, while only $20 \%$ of the visits to private GP clinics were for the same. ${ }^{20}$ The main reason for the high utilisation of public primary care for chronic diseases is because of the availability of government subsidy. The average consultation fee borne by patients per visit is SGD13.20 and SGD20-55 in the polyclinic (after government subsidy) and private GP clinic, respectively. ${ }^{15}$

\section{Study population}

National Healthcare Group (NHG) is 1 of the 3 integrated healthcare clusters, and serves the central region of Singapore. ${ }^{21}$ National Healthcare Group Polyclinics (NHGP) is the primary care arm of NHG that serves the population in the central and northern regions of Singapore.

NHGP maintains an administrative database on all patients who seek treatment at any of its polyclinics. Data such as patient demographics, chronic diseases and polyclinic service utilisation codes were extracted from patient electronic medical records kept in NHGP's administrative database. International Statistical Classification of Disease and Related Health Problems, 10th Revision (ICD-10) 22 was used to capture all medical diagnoses. De-identified data were examined for this study. Ethics Review Committee approval was obtained with waiver of patient consent (DSRB reference 2018/01164). 
For this retrospective cohort study, we included all Singaporeans and permanent residents from 21 to 99 years old who had at least 1 chronic disease. We selected patients who visited the polyclinic at least twice from July 2015 to June 2016 (as a proxy to indicate that they were with the healthcare setting regularly for their chronic diseases) and at least once from July 2016 to June 2017 (as a proxy that they were still on follow-up with the healthcare setting). All patients who were deceased within the study period were excluded (Fig. 1).

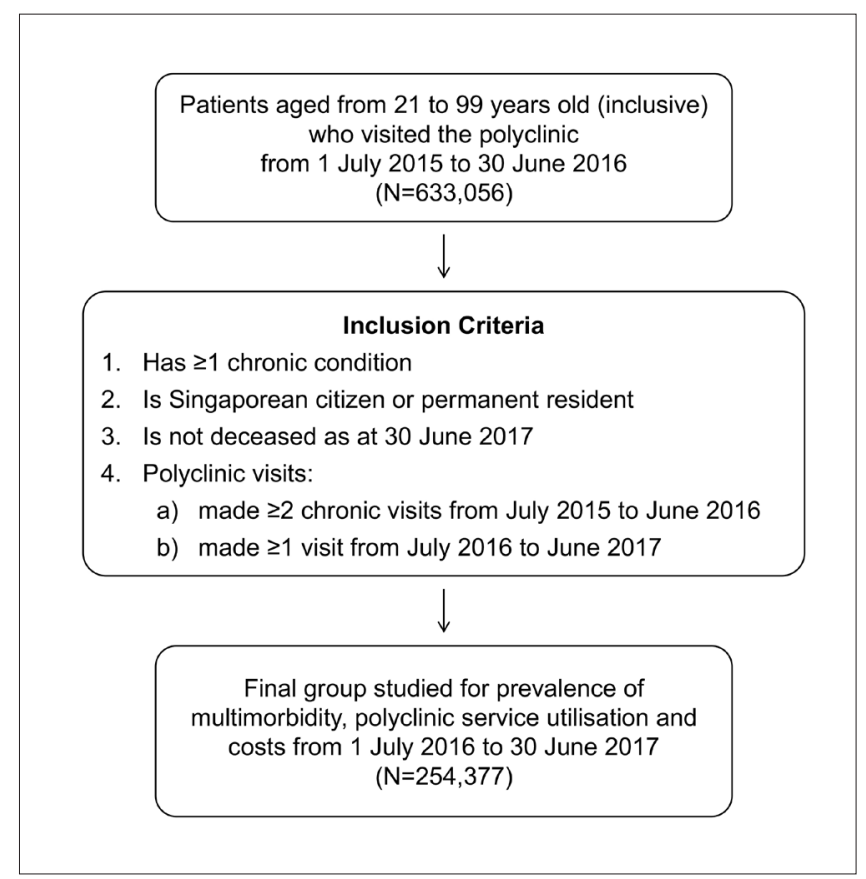

Fig. 1. Flow diagram for selection of patients.

\section{Study variables}

We selected chronic diseases based on the list proposed by Fortin et al. who shortlisted 20 diseases that were relevant to multimorbidity based on their prevalence and clinical significance. ${ }^{23}$ Four family physicians in NHGP reviewed the ICD-10 codes and matched 39 of them to the Fortin's list of 20 chronic medical diseases. The ICD codes were reviewed based on a 4-step approach proposed by N'Goran et al. ${ }^{24}$ However, one of the 20 diseases-back pain-did not have a compatible ICD-10 code as the presentation of back pain in the polyclinic practice setting is often coded as an acute medical condition. Hence, the final number of chronic diseases for the multimorbidity list was reduced to 19 (Table S1 of Supplementary Appendix in the online version of this article).
Every selected patient was coded for the presence or absence of any of the 19 chronic diseases. We defined multimorbidity as having at least 3 or more chronic diseases. $^{5}$

\section{Demographic variables}

The demographic data collected included age, sex, ethnicity (Chinese, Malay, Indian and others) and residential status (Singaporean and permanent resident).

\section{Healthcare cost}

Healthcare cost is defined as the gross charges incurred by the patient before any government subsidy from July 2016 to June 2017. Only cost incurred from utilisation of services in NHGP were included. These services included consultations with doctors, nurses or allied healthcare professionals, as well as laboratory, radiological, screening, immunisation and pharmacy services. Dental services were not included. The total costs incurred by patients who visited NHGP for management of their chronic medical diseases were presented as annual healthcare costs per capita in Singapore dollar (SGD), rounded to the nearest dollar.

\section{Statistical analysis}

Descriptive statistics were calculated including frequencies, percentages, means and standard deviations, or medians and interquartile ranges. The Mann-Whitney $\mathrm{U}$ test (Table 3) was used to compare median annual cost per capita incurred by patients with multimorbidity and those without multimorbidity for each demographic sub-group. Data cleaning and tabulation of descriptive statistics were conducted using Python software version 3.7.7 (Python Software Foundation) while statistical analyses were conducted using $\mathrm{R}$ software version 3.6.3. ${ }^{25}$ Statistical significance was set as $P<0.05$.

\section{RESULTS}

There were 254,377 patients in our study population (Fig. 1). Table 1 shows that the largest proportion of patients had 3 chronic diseases $(26.0 \%)$. The study population comprised $52.8 \%$ female. The sex ratio of 1:1 remained very similar for increasing number of chronic diseases. The majority of patients were Chinese $(76.9 \%)$, followed by Malay (11.4\%), Indian $(8.7 \%)$ and others $(3.0 \%)$. The highest proportion of patients who had 1 chronic disease was from the others ethnicity $(17.6 \%)$. Patients from the Chinese ethnic group had the highest proportion for $2(24.0 \%)$ and $3(26.6 \%)$ chronic diseases. Patients from the Malay ethnic group had the highest proportion for 4 chronic 
Table 1. Proportions and mean number of medical diseases by baseline characteristics

\begin{tabular}{|c|c|c|c|c|c|c|}
\hline & \multirow{2}{*}{$\begin{array}{c}\mathrm{N}=254,377 \\
\text { No. of patients (\%) }\end{array}$} & \multicolumn{5}{|c|}{ Number of chronic diseases } \\
\hline & & 1 & 2 & 3 & 4 & $\geq 5$ \\
\hline & & 14.8 & 22.8 & 26.0 & 20.0 & 16.4 \\
\hline \multicolumn{7}{|l|}{ Sex } \\
\hline Female & $134,430(52.8)$ & 15.5 & 23.1 & 25.6 & 19.6 & 16.2 \\
\hline Male & $119,947(47.2)$ & 14.1 & 22.6 & 26.4 & 20.4 & 16.6 \\
\hline \multicolumn{7}{|c|}{ Ethnicity } \\
\hline Chinese & $195,581(76.9)$ & 15.0 & 24.0 & 26.6 & 19.4 & 15.0 \\
\hline Malay & $29,049(11.4)$ & 14.7 & 18.5 & 24.0 & 22.3 & 20.5 \\
\hline Indian & $22,007(8.7)$ & 12.8 & 18.8 & 23.6 & 22.1 & 22.7 \\
\hline Others & $7,740(3.0)$ & 17.6 & 22.1 & 23.6 & 19.6 & 17.1 \\
\hline \multicolumn{7}{|c|}{ Age group, years } \\
\hline $21-30$ & $3,818(1.5 \%)$ & 71.9 & 19.0 & 5.7 & 2.7 & 0.8 \\
\hline $31-40$ & $6,725(2.6)$ & 43.9 & 27.8 & 16.3 & 8.5 & 3.5 \\
\hline $41-50$ & $24,818(9.8)$ & 27.8 & 29.6 & 22.3 & 12.9 & 7.4 \\
\hline $51-60$ & $68,644(27.0)$ & 17.7 & 27.0 & 26.3 & 17.5 & 11.5 \\
\hline $61-70$ & $85,624(33.7)$ & 11.1 & 23.1 & 28.5 & 21.2 & 16.1 \\
\hline $71-80$ & $45,528(17.9)$ & 6.2 & 16.4 & 27.0 & 25.4 & 25.0 \\
\hline$>80$ & $19,220(7.6)$ & 3.8 & 12.3 & 23.3 & 26.7 & 33.9 \\
\hline
\end{tabular}

diseases $(22.3 \%)$ and the Indian ethnic group had the highest proportion for 5 or more chronic diseases (22.7\%). Majority of the patients were in the age range of $61-70$ years $(33.7 \%)$. The highest proportion of patients with 1 chronic disease was the 21-30 years age group $(71.9 \%)$, while the highest proportion of patients with 5 or more chronic diseases were from the $>80$ years age group (33.9\%).

Table 2 shows the annual cost per capita by number of chronic diseases. The median annual cost per capita increased with the number of chronic diseases from 1 chronic disease of SGD277 to 5 or more chronic diseases of SGD895 (USD1 $=$ SGD1.38 as of 30 June $2017^{26}$ ). The greatest percentage increment in median cost was from 2 to 3 chronic diseases (an increment of $43.0 \%)$.

Up to $76.6 \%$ of the annual healthcare cost was incurred by patients with multimorbidity (3 or more diseases) who made up $62.4 \%$ of the study population (Fig. 2).

Table 3 shows the difference in annual cost per capita in patients with and without multimorbidity. The median annual healthcare cost per capita for patients

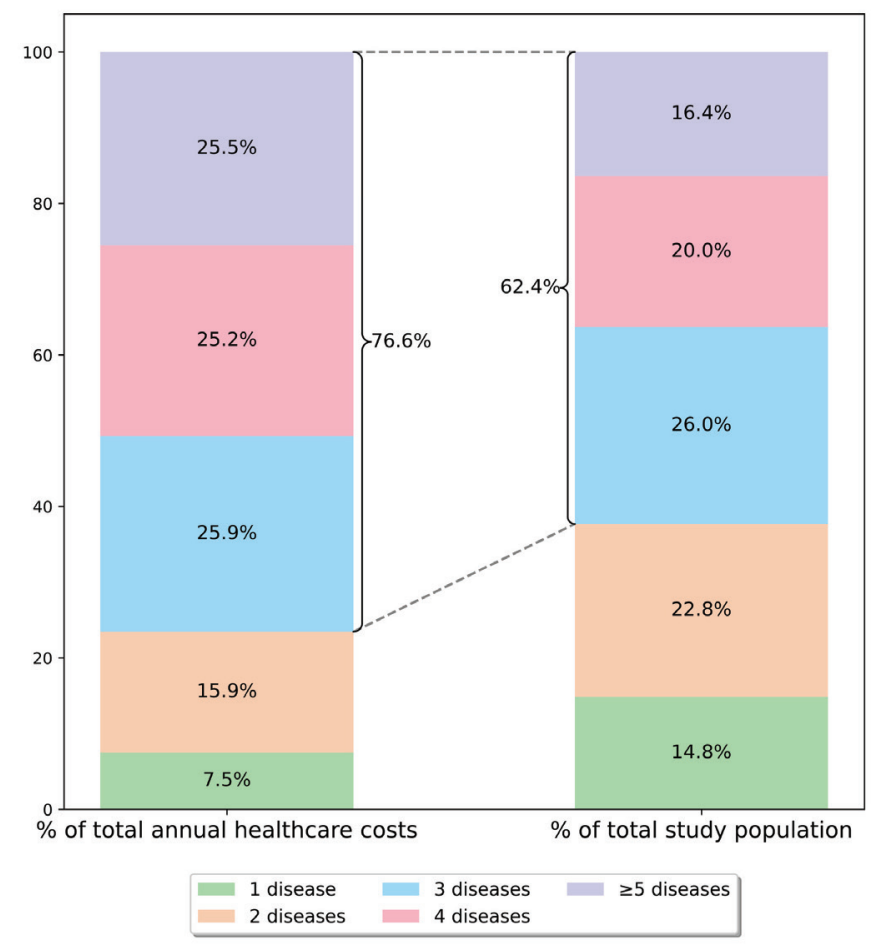

Fig. 2 Proportion of annual healthcare costs incurred by proportion of study population. 
Table 2. Annual cost per capita (SGD) by number of chronic diseases

\begin{tabular}{lccccc}
\hline & & \multicolumn{3}{c}{ Annual cost per capita } \\
\cline { 3 - 5 } $\begin{array}{l}\text { No. of } \\
\text { diseases }\end{array}$ & $\begin{array}{c}\text { N=254,377 } \\
\text { No. of patients (\%) }\end{array}$ & Mean (SD) & $\begin{array}{c}\text { Incremental mean cost } \\
\text { per increase in disease } \\
\text { (\% change) }\end{array}$ & Median (IQR) & $\begin{array}{c}\text { Incremental median cost } \\
\text { per increase in disease } \\
\text { (\% change) }\end{array}$ \\
\hline 1 & $37,773(14.8)$ & $332(256)$ & NA & $277(168-427)$ & NA \\
\hline 2 & $58,092(22.8)$ & $457(312)$ & $125(37.6)$ & $388(262-571)$ & $112(40.4)$ \\
\hline 3 & $66,093(26.0)$ & $652(434)$ & $195(42.7)$ & $555(373-809)$ & $167(43.0)$ \\
\hline 4 & $50,776(20.0)$ & $827(521)$ & $175(26.9)$ & $716(483-1,030)$ & $161(28.9)$ \\
\hline$\geq 5$ & $41,643(16.4)$ & $1,021(619)$ & $194(23.4)$ & $895(606-1,292)$ & $180(25.1)$ \\
\hline
\end{tabular}

IQR: interquartile range; NA: not applicable; SD: standard deviation

${ }^{\text {a }}$ Figures are rounded to the nearest dollar

Table 3. Annual cost per capita (SGD) by baseline characteristics and multimorbidity ${ }^{\mathrm{a}}$ status

\begin{tabular}{|c|c|c|c|c|c|c|}
\hline & \multicolumn{3}{|c|}{ Without multimorbidity } & \multicolumn{3}{|c|}{ With multimorbiditya } \\
\hline & \multirow[t]{2}{*}{ No. $(\%)$} & \multicolumn{2}{|c|}{ Annual cost per capita } & \multirow[t]{2}{*}{ No. $(\%)$} & \multicolumn{2}{|c|}{ Annual cost per capita ${ }^{b}$} \\
\hline & & Mean (SD) & Median (IQR) & & Mean (SD) & Median (IQR) \\
\hline & $\mathrm{n}=95,865$ & 408 (297) & $344(219-518)$ & $\mathrm{n}=158,512$ & 805 (537) & $683(446-1,013)$ \\
\hline \multicolumn{7}{|l|}{ Sex } \\
\hline Female & $51,896(54.1)$ & $394(277)$ & $335(216-502)$ & $82,534(52.1)$ & $793(525)$ & $672(441-995)$ \\
\hline Male & $43,969(45.9)$ & $424(319)$ & $354(223-537)$ & 75,978 (47.9) & $819(549)$ & $695(452-1,033)$ \\
\hline \multicolumn{7}{|l|}{ Ethnicity } \\
\hline Chinese & $76,192(79.5)$ & $399(278)$ & $340(221-505)$ & $119,389(75.3)$ & $775(510)$ & $660(435-972)$ \\
\hline Malay & $9,650(10.1)$ & $433(350)$ & $354(208-563)$ & $19,399(12.2)$ & $892(593)$ & $756(490-1,137)$ \\
\hline Others & $3,071(3.2)$ & $427(347)$ & 345 (207-549) & $4,669(2.9)$ & $835(567)$ & $714(451-1,058)$ \\
\hline \multicolumn{7}{|c|}{ Age group, years } \\
\hline $21-30$ & $3,469(3.6)$ & $344(358)$ & $249(117-446)$ & $349(0.2)$ & $593(528)$ & $455(219-791)$ \\
\hline $31-40$ & $4,819(5.0)$ & $383(307)$ & $314(179-496)$ & $1,906(1.2)$ & $725(520)$ & $616(372-933)$ \\
\hline $41-50$ & $14,237(14.9)$ & $401(309)$ & $330(202-514)$ & $10,581(6.7)$ & $764(558)$ & $632(401-954)$ \\
\hline $51-60$ & $30,701(32.0)$ & 404 (299) & $336(216-509)$ & $37,943(23.9)$ & $791(552)$ & 655 (425-987) \\
\hline $61-70$ & $29,273(30.5)$ & $411(279)$ & $351(231-516)$ & $56,351(35.5)$ & $813(543)$ & $685(449-1,023)$ \\
\hline $71-80$ & $10,277(10.7)$ & $440(293)$ & $380(256-555)$ & $35,251(22.2)$ & $829(522)$ & $720(478-1,045)$ \\
\hline$>80$ & $3,089(3.2)$ & $459(295)$ & $397(265-592)$ & $16,131(10.2)$ & $800(493)$ & $704(470-1,013)$ \\
\hline
\end{tabular}

IQR: interquartile range; SD: standard deviation

${ }^{a}$ Multimorbidity is defined as 3 or more chronic diseases

${ }^{\mathrm{b}}$ Figures are rounded to the nearest dollar. Mann-Whitney $\mathrm{U}$ tests were conducted for each horizontal row and all were statistically significant at $P<0.001$

with multimorbidity was about twice that for patients without multimorbidity (SGD683 vs SGD344). Patients with multimorbidity incurred a higher median annual cost per capita compared to those without multimorbidity across all sex, ethnic and age groups. These costs were also approximately twice the amount incurred by patients without multimorbidity.

Table 4 shows the most prevalent triads ( 3 chronic diseases) and their annual cost per capita. The median annual cost per capita for these top 10 triads ranged 
from SGD418 (hyperlipidaemia, hypertension and obesity) to SGD747 (hyperlipidaemia, hypertension and type 2 diabetes mellitus). Besides being the most expensive triad, "hyperlipidaemia, hypertension and type 2 diabetes mellitus" as a triad was also the most prevalent $(31.0 \%)$. This is followed by the triad of "hyperlipidaemia, hypertension, and arthritis and/or rheumatoid arthritis" (11.4\%); and "hyperlipidaemia, hypertension and cardiovascular disease" (6.3\%). The median annual cost per capita for the second and third most prevalent triad was SGD439 and SGD571, respectively.

\section{DISCUSSION}

Our study showed that the prevalence of multimorbidity in the study population who had at least 1 chronic disease was $62.4 \%$. The median annual healthcare cost per capita increased with the number of chronic diseases from 1 chronic disease of SGD277 to more than 5 chronic diseases of SGD895. The greatest percentage increment in cost was from 2 to 3 chronic diseases. Patients with multimorbidity incurred a higher median annual cost per capita compared to those without multimorbidity across all sex, ethnic and age groups. The median annual healthcare cost per capita, for patients with multimorbidity was about twice that for patients without multimorbidity
(SGD683 vs SGD344). The cost for the most prevalent 10 triads ranged from SGD418 (hyperlipidaemia, hypertension and obesity) to SGD747 (hyperlipidaemia, hypertension and type 2 diabetes mellitus). The most prevalent triad "hyperlipidaemia, hypertension and type 2 diabetes mellitus" was also the most expensive triad.

It is not surprising that the triad of hypertension, hyperlipidaemia and diabetes is the most prevalent. Based on the National Primary Care Survey 2014, ${ }^{20}$ these 3 diseases are among the top 5 principal diagnoses in the polyclinics. This will also explain why these diseases are highly represented in the other triads shown in Table 4.

The most prevalent triad of hypertension, hyperlipidaemia and diabetes is also most expensive due to increased healthcare utilisation. A typical patient with previously well-controlled hypertension and hyperlipidaemia when diagnosed with diabetes would have to take new medication for diabetes. In addition, there will be extra testing of $\mathrm{HbAlc}$ on a regular basis, annual renal and urine test, as well as annual eyes and feet screening. The increased healthcare costs incurred in primary care for treatment and monitoring are necessary to prevent further complications of diabetes.

We noted that the Malay and Indian ethnic groups had a higher proportion representation in the multimorbidity

Table 4. Top 10 triads (by prevalence) and their annual cost per capita (SGD)

\begin{tabular}{|c|c|c|c|c|c|c|}
\hline \multirow[t]{2}{*}{ Rank } & \multirow[t]{2}{*}{ Disease 1} & \multirow[t]{2}{*}{ Disease 2} & \multirow[t]{2}{*}{ Disease 3} & \multirow{2}{*}{$\begin{array}{c}\text { No. of } \\
\text { patients }(\%)\end{array}$} & \multicolumn{2}{|c|}{ Annual cost per capita ${ }^{a}$} \\
\hline & & & & & Mean (SD) & Median (IQR) \\
\hline 1 & Hyperlipidaemia & Hypertension & Type 2 diabetes mellitus & $20,499(31.0)$ & $864(511)$ & $747(533-1,052)$ \\
\hline 2 & Hyperlipidaemia & Hypertension & $\begin{array}{c}\text { Arthritis and/or rheumatoid } \\
\text { arthritis }\end{array}$ & $7,553(11.4)$ & $481(248)$ & $439(311-603)$ \\
\hline 3 & Hyperlipidaemia & Hypertension & $\begin{array}{l}\text { Cardiovascular disease } \\
\text { (angina, myocardial infarct, atrial } \\
\text { fibrillation, poor circulation } \\
\text { of lower limbs) }\end{array}$ & $4,158(6.3)$ & $625(327)$ & $571(412-780)$ \\
\hline 4 & Hyperlipidaemia & Hypertension & Obesity & $3,836(5.8)$ & $456(222)$ & $418(304-563)$ \\
\hline 5 & Hyperlipidaemia & Hypertension & $\begin{array}{l}\text { Stroke and transient } \\
\text { ischaemic attack }\end{array}$ & $2,539(3.8)$ & $560(284)$ & $518(373-698)$ \\
\hline 6 & Hyperlipidaemia & Hypertension & Chronic hepatitis & $1,878(2.8)$ & $471(230)$ & $430(311-592)$ \\
\hline 7 & Hyperlipidaemia & $\begin{array}{l}\text { Type } 2 \text { diabetes } \\
\text { mellitus }\end{array}$ & $\begin{array}{l}\text { Arthritis and/or } \\
\text { rheumatoid arthritis }\end{array}$ & $1,759(2.7)$ & $722(438)$ & $622(447-876)$ \\
\hline 8 & Hyperlipidaemia & Hypertension & Kidney disease or failure & $1,345(2.0)$ & $532(283)$ & $481(346-672)$ \\
\hline 9 & Hyperlipidaemia & Hypertension & Thyroid disorder & $1,269(1.9)$ & $530(245)$ & $489(354-662)$ \\
\hline 10 & Hyperlipidaemia & $\begin{array}{l}\text { Type } 2 \text { diabetes } \\
\text { mellitus }\end{array}$ & Obesity & $1,230(1.9)$ & $774(510)$ & $651(452-946)$ \\
\hline
\end{tabular}

IQR: interquartile range; SD: standard deviation

${ }^{\text {a }}$ Figures are rounded to the nearest dollar 
group. This finding was similar to a previous report by Subramaniam et al. ${ }^{3}$ where the risk of having 2 of more chronic diseases was higher in the Malay ethnic group after controlling for sex.

Although healthcare cost increased with the number of chronic diseases, the incremental cost was non-linear (Table 2). The increase in healthcare cost with number of chronic diseases was also reported in a local study conducted in 2012-2013 by Picco et al. ${ }^{13}$ The authors estimated that the primary healthcare cost for no chronic disease, 1 chronic disease, 2 or more chronic diseases were SGD81, SGD202 and SGD303, respectively. The incremental amount from no to 1 chronic disease was SGD121, and SGD101 from 1 to 2 chronic diseases. The non-linear incremental cost with increase in chronic diseases was also reported in a US study. The study found that the percentage change was highest from 2 to 3 diseases at $33 \%$ and only $18 \%$ when diseases increased from 10 to $11 .{ }^{27}$

Further study is needed to better understand what the drivers of the incremental cost are as the number of chronic diseases increases. These could be factors such as pattern of multimorbidity, disease trajectory, stages of disease, complications of diseases, intensity of monitoring and treatment (polypharmacy), intrinsic biopsychosocial reserve, and differential cost loading between primary and tertiary care.

We postulated that the pattern of multimorbidity may be one of the significant cost drivers. The pattern of multimorbidity in our primary care population showed a high prevalence and strong tendency of clustering cardiometabolic diseases such as hyperlipidaemia, hypertension, type 2 diabetes, cardiovascular and cerebrovascular diseases. Another published article using statistical methods to identify systematic clusters among diseases (i.e. associative multimorbidity) also identified cardiometabolic diseases as a prominent pattern. ${ }^{28} \mathrm{~A}$ reason for their high prevalence in primary care is these cardiometabolic chronic diseases have a relatively long asymptomatic trajectory (years) before any manifestation of end-organ damage, and patients are thus cared for in primary care for a long period. Other than being highly prevalent, this pattern of multimorbidity also incurred the highest healthcare cost in our study. Pattern of multimorbidity underpins the interaction between diseases, the treatment burden and hence influences healthcare cost. Disease that are concordant in nature, sharing similar pathophysiology and synergistic management approach, are usually managed in the primary care setting (e.g. cardiometabolic diseases). These diseases usually received substantial drug subsidy and there are welldeveloped guidelines to manage these diseases in the primary healthcare setting. This may explain the relatively high healthcare cost of such diseases in the primary care setting. Diseases that are discordant in their pathophysiology, which may require more complicated management approach, are likely to have their cost transferred to tertiary care.

Our findings allowed us to appreciate the direct and incremental public primary care healthcare cost of multimorbidity incurred in a developed multiethnic population such as Singapore. There are many other challenges of multimorbidity, besides healthcare cost. Current indicators for quality of care are largely targeted at single diseases with little consideration of the interaction between the myriad of chronic diseases and each patient's unique constitutions. ${ }^{29}$ Evidence suggests that adherence to single disease guidelines will lead to polypharmacy. ${ }^{30}$ To date, there are no conclusive evidence for effective interventions in the management of multimorbidity although intervention targeted at mental health had some positive results. ${ }^{31,32}$ We advocate that one main focus in the management of patients with chronic disease(s) should be on the prevention or delay in the onset of other chronic diseases. Attention to reduce the incidence of multimorbidity and research agendas have also been proposed in recent literature on multimorbidity. ${ }^{33,34}$

\section{Strengths and limitations}

The strength of our study included the use of a validated and extensive list of common chronic diseases in the definition of multimorbidity. We also used direct cost data from the public primary care provider, which provided a more accurate healthcare utilisation estimate. The healthcare cost presented is a good estimate for allocable government cost. This estimate is also not affected by deductibles and co-payments by insurance. However, patients' out-of-pocket expenses for nonsubsidised medication is not reflected in our cost.

There are 4 main limitations in this study. Firstly, like most research related to the use of electronic medical records, this study was subjected to possible disease coding error from either system or provider limitations. Secondly, this study could be strengthened by considering the severity of each chronic disease. Thirdly, we are unable to generalise our finding to what the total healthcare cost is to the entire system as cost incurred by utilisation of other private primary care (e.g. 
general practitioner clinics), hospitals and community services (e.g. nursing homes) were not available. Finally, further analytical examination could be undertaken to uncover which additional chronic disease contributes the most to the incremental healthcare cost.

\section{CONCLUSION}

Multimorbidity is an important public healthcare issue in developed countries grappling with an ageing population such as Singapore. There is a great need to improve our current understanding of multimorbidity in its multi-faceted domains. This study hopes to contribute to the understanding in terms of the cost and pattern of multimorbidity in a public primary care setting.

\section{Funding}

This research was supported by the Singapore Ministry of Health's National Medical Research Council under the Centre Grant Programme (reference number: $N M R C / C G / C 019 / 2017$ ).

\section{REFERENCES}

1. Fortin M, Stewart M, Poitras ME, et al. A systematic review of prevalence studies on multimorbidity: Toward a more uniform methodology. Ann Fam Med 2012;10:142-51.

2. Quah JHM, Wang P, Ng RRG, et al. Health-related quality of life of older asian patients with multimorbidity in primary care in a developed nation. Geriatr Gerontol Int 2017;17:1429-37.

3. Subramaniam M, Abdin E, Picco L, et al. Multiple chronic medical conditions: Prevalence and risk factors - results from the Singapore mental health study. Gen Hosp Psychiatry 2014; 36:375-81

4. Low LL, Kwan YH, Ko MSM, et al. Epidemiologic characteristics of multimorbidity and sociodemographic factors associated with multimorbidity in a rapidly aging asian country. JAMA Netw Open 2019;2:e1915245.

5. Lee YAJ, Xie Y, Lee PSS, et al. Comparing the prevalence of multimorbidity using different operational definitions in primary care in Singapore based on a cross-sectional study using retrospective, large administrative data. BMJ Open 2020;10:e039440.

6. Civil Service College, Singapore. Successful ageing: Progressive governance and collaborative communities, 6 July 2018. Available at: https://www.csc.gov.sg/articles/successful-ageing-progressivegovernance-and-collaborative-communities. Accessed on 19 August 2021.

7. Bahler C, Huber CA, Brungger B, et al. Multimorbidity, health care utilization and costs in an elderly community-dwelling population: A claims data based observational study. BMC Health Serv Res 2015;15:23.

8. Orueta JF, García-Álvarez A, García-Goñi M, et al. Prevalence and costs of multimorbidity by deprivation levels in the basque country: A population based study using health administrative databases. PLoS One 2014;9:e89787.

9. Frølich A, Ghith N, Schiøtz M, et al. Multimorbidity, healthcare utilization and socioeconomic status: A register-based study in denmark. PLoS One 2019;14:e214183.
10. Glynn LG, Valderas JM, Healy P, et al. The prevalence of multimorbidity in primary care and its effect on health care utilization and cost. Fam Pract 2011;28:516-23.

11. Thavorn K, Maxwell CJ, Gruneir A, et al. Effect of sociodemographic factors on the association between multimorbidity and healthcare costs: A population-based, retrospective cohort study. BMJ Open 2017;7:e017264.

12. Ministry of Finance, Singapore. Injecting more into Singapore's healthcare as we age, 20 October 2020. Available at: https://www. mof.gov.sg/singapore-budget/curated-budget-reads/. Accessed on 19 August 2021.

13. Picco L, Achilla E, Abdin E, et al. Economic burden of multimorbidity among older adults: Impact on healthcare and societal costs. BMC Health Serv Res 2016;16:173.

14. Lee ES, Lee PSS, Xie Y, et al. The prevalence of multimorbidity in primary care: A comparison of two definitions of multimorbidity with two different lists of chronic conditions in Singapore. BMC Public Health 2021;21:1409.

15. Lee CE. International health care system profiles (Singapore), 5 June 2020. Available at: https://www.commonwealthfund.org/internationalhealth-policy-center/countries/singapore\#universal-coverage. Accessed on 27 Oct 2020.

16. Ministry of Health, Singapore. Primary healthcare services, 19 April 2020. Available at: https://www.moh.gov.sg/home/our-healthcaresystem/healthcare-services-and-facilities/primary-healthcare-services. Accessed on 18 October 2020

17. Khoo HS, Lim YW, Vrijhoef HJM. Primary healthcare system and practice characteristics in Singapore. Asia Pac Fam Med 2014;13:8.

18. Tan KB, Earn Lee C. Integration of primary care with hospital services for sustainable universal health coverage in Singapore. Health Syst Reform 2019;5:18-23.

19. Ministry of Health, Singapore. Admissions and outpatient attendances 2018-2020, 31 May 2021. Available at: https://www.moh. gov.sg/resources-statistics/singapore-health-facts/admissions-andoutpatient-attendances. Accessed on 19 August 2021.

20. Ministry of Health, Singapore. Primary care survey 2014 report, 2014. Available at: https://www.moh.gov.sg/resources-statistics/reports/ primary-care-survey-2014-report. Accessed on 18 October 2020

21. National Healthcare Group. NHG profile, 19 October 2020. Available at: https://corp.nhg.com.sg/AboutNHG/Pages/NHG-Profile. aspx. Accessed on 27 October 2020

22. World Health Organization. International statistical classification of diseases and related health problems 10th revision, 2010. Available at: https://icd.who.int/browse10/2010/en. Accessed on 27 October 2020.

23. Fortin M, Almirall J, Nicholson K. Development of a research tool to document self-reported chronic conditions in primary care. J Comorb 2017;7:117-23.

24. N'Goran AA, Blaser J, Deruaz-Luyet A, et al. From chronic conditions to relevance in multimorbidity: A four-step study in family medicine. Fam Pract 2016;33:439-44.

25. $\mathrm{R}$ Core Team. R: A language and environment for statistical computing. Vienna, Austria: R Foundation for Statistical Computing; 2017.

26. OANDA Corporation. Currency converter, 1996. Available at: https://www1.oanda.com/currency/converter/. Accessed on 23 August 2021.

27. Hajat C, Siegal Y, Adler-Waxman A. Clustering and healthcare costs with multiple chronic conditions in a us study. Front Public Health 2020;8:607528. 
28. Tan XW, Xie Y, Lew JK, et al. Patterns of patients with multiple chronic conditions in primary care: A cross-sectional study. PLoS One 2020;15:e0238353.

29. Ong KY, Lee PSS, Lee ES. Patient-centred and not disease-focused: A review of guidelines and multimorbidity. Singapore Med J 2020;61:584-90.

30. Boyd CM, Darer J, Boult C, et al. Clinical practice guidelines and quality of care for older patients with multiple comorbid diseases: Implications for pay for performance. Jama 2005; 294:716-24.

31. Smith SM, Wallace E, O'Dowd T, et al. Interventions for improving outcomes in patients with multimorbidity in primary care and community settings. Cochrane Database Syst Rev 2021;1:CD006560.

32. Contant E, Loignon C, Bouhali $\mathrm{T}$, et al. A multidisciplinary self-management intervention among patients with multimorbidity and the impact of socioeconomic factors on results. BMC Fam Pract 2019;20:53.

33. Head A, Fleming K, Kypridemos C, et al. Multimorbidity: The case for prevention. J Epidemiol Community Health 2021;75:242-4.

34. Pearson-Stuttard J, Ezzati M, Gregg EW. Multimorbidity-a defining challenge for health systems. Lancet Public Health 2019;4:e599-e600. 